This peer reviewed manuscript has been accepted for publications to the Tribology Letters. Cite this article as: Luis Bartolomé, Eva Oblak, Mitjan Kalin, Sliding evolution of the mechanical behaviour of zinc dialkyldithiophosphate tribofilms on diamond-like carbon coatings Tribology letters 62 (2016) 01-09. DOI: https://doi.org/ 10.1007/s11249-016-0666-y

\title{
Sliding evolution of the mechanical behaviour of zinc dialkyldithiophosphate tribofilms on diamond-like carbon coatings
}

\author{
Luis Bartolomé, Eva Oblak, Mitjan Kalin
}

Laboratory for Tribology and Interface Nanotechnology, Faculty of Mechanical Engineering, University of Ljubljana, Bogišićeva 8, 1000 Ljubljana, Slovenia *Corresponding author: luis.bartolome@tint.fs.uni-lj.si

Keywords: DLC, ZDDP, Finite-element method, AFM

The evolution of the mechanical behaviour of zinc dialkyldithiophosphate (ZDDP) tribofilms on diamond-like carbon (DLC) coatings during sliding contact has been studied by combining nano-indentation experimental data and finite element modelling. The nano-indentation data from the ZDDP tribofilms were obtained for two different sliding distances of the tribological tests after 15,000 and 216,000 cycles, which correspond to 25 minutes and 6 hours respectively. A non-homogeneous structure was found for the ZDDP tribofilms after both sliding distances, which were also verified by the local stiffness using the AFM forcemodulation mode. A dual structure is observed for the ZDDP tribofilms after 15,000 cycles, while three distinct components with different mechanical behaviours were found after 216,000 cycles. It was also found that the mechanical behaviour of the harder component of the ZDDP tribofilms remains almost unchanged for both sliding times, while the softer component tends to stiffen slightly over the sliding time. In addition, using the proposed algorithm with finite element simulations, the mechanical properties of the different components have been obtained, e.g. the elastic modulus $E=25.5 \mathrm{GPa}$ and the yield stress $Y=1.585 \mathrm{GPa}$ of the harder component for both sliding times. The softer component exhibits $E=1.65 \mathrm{GPa}$ and $Y=0.144 \mathrm{GPa}$ after 15,000 cycles and $E=3.5 \mathrm{GPa}$ and $Y=0.17 \mathrm{GPa}$ after 216,000 cycles. Finally, an intermediate component with $E=11.7 \mathrm{GPa}$ and $Y=0.94 \mathrm{GPa}$ has been only found at 216,000 cycles.

\section{INTRODUCTION}

Surface engineering technologies have seen a significant progress over the past decade. The application of thin coatings, such as DLC, on the surfaces of engine components has provided significant improvements for the automotive industry, especially in terms of decreasing fuel consumption due to energy losses. DLC coatings lead to a reduction in friction, particularly in poor lubrication conditions. DLC coatings also provide an improvement in wear resistance due to their high hardness, high corrosion resistance and chemical stability [1]. In spite of contrasting results, especially early on, in boundary lubrication conditions, DLC coatings also form tribofilms, which help in reducing both wear and friction [2]. 
This peer reviewed manuscript has been accepted for publications to the Tribology Letters. Cite this article as: Luis Bartolomé, Eva Oblak, Mitjan Kalin, Sliding evolution of the mechanical behaviour of zinc dialkyldithiophosphate tribofilms on diamond-like carbon coatings Tribology letters 62 (2016) 01-09. DOI: https://doi.org/ 10.1007/s11249-016-0666-y

Among the various automotive additives, ZDDP is one of the most indispensable and crucial due to its excellent anti-wear properties. ZDDP tribofilms have been widely studied for tribofilms formed on steel surfaces [2, 3], but less so on DLC coatings [4, 5]. Some recent analyses of the mechanical properties and morphology revealed the complexity of ZDDP tribofilms, depending on the DLC coating and the lubricant's additives [6-8]. This complexity in structure has also been observed in molecular dynamics simulations of ZDDP tribofilms

[9]. In our earlier work, we studied the behaviour for a specific testing time [10]. However, how the structure of ZDDP tribofilms and the mechanical behaviour of their components evolve during the sliding time has not been studied in depth.

Knowledge of the mechanical behaviour of the different components of ZDDP tribofilms is needed to understand the origin and the effects on friction and wear, but also in numerical simulations to predict the deformation at contacts and modelling both friction and wear. Therefore, this work has focused on the sliding evolution of the mechanical behaviour of ZDDP tribofilms formed in boundary lubricated conditions on DLC coatings.

In order to establish the constitutive models for the ZDDP tribofilms, the experimental data were obtained from nano-indentations which have been widely used for studying the mechanical properties of engineering materials, coatings and films [11-16]. The methods used to extract the mechanical properties from the load-displacement curves of nano-indentations can be divided into two main categories [11]. The first category uses loading-unloading curves to extract the mechanical properties, extending the classic elastic solution of an infinite half space, e.g. the method introduced by Oliver and Pharr [12]. The second category uses intensive FE simulations for generating load-displacement curves for various combinations of mechanical properties and then trying to match the experimental data of the unknown material with the numerical results from the FE simulations to extract the mechanical properties of the analysed material [13-16].

In this work, the experimental loading curves of Berkovich indentations were used for establishing how the mechanical behaviour of the ZDDP tribofilms formed on DLC coatings evolves over time. In order to extract the mechanical properties from the experimental nanoindentation data, an algorithm, which consists of a forward algorithm with intensive finite element (FE) simulations and of an inverse algorithm for fitting the FE results of the forward algorithm, has been proposed. Therefore, the analysis of the constitutive models for ZDDP tribofilms is based on methods from the second category, which was found to give better results [17]. Using this algorithm, the constitutive model that best fits the mechanical behaviour of the ZDDP tribofilms is obtained, as well as the mechanical properties of the ZDDP tribofilms. It is interesting that two different components are found for ZDDP tribofilms obtained after a short time [10], while long term results show up to three components. 
This peer reviewed manuscript has been accepted for publications to the Tribology Letters. Cite this article as: Luis Bartolomé, Eva Oblak, Mitjan Kalin, Sliding evolution of the mechanical behaviour of zinc dialkyldithiophosphate tribofilms on diamond-like carbon coatings Tribology letters 62 (2016) 01-09. DOI: https://doi.org/ 10.1007/s11249-016-0666-y

\section{METHODOLOGY}

\subsection{Experimental methods}

In order to obtain the ZDDP tribofilms for the nano-indentation, tribological tests were carried out using a tribometer (UMT-2, CETR, CA, USA). The test configuration was a ball on flat in reciprocating motion. The steel balls were rubbed against H-DLC coated discs that were immersed in a solution of base oil (Group III, Lubrizol, OH, USA) suitable for automotive applications, with $1 \mathrm{wt} \%$ of ZDDP (Lubrizol, OH, USA) providing the ZDDP tribofilms on the DLC coatings. The H-DLC coating is a conventionally hydrogenated amorphous DLC coating with an $\mathrm{sp} 3 /(\mathrm{sp} 3+\mathrm{sp} 2)$ ratio from $35 \%$ to $40 \%$ and a hydrogen content of 30 at. $\%$. The thickness of the H-DLC coating was $0.8 \mu \mathrm{m}$, with two additional interlayers; a Ti-based interlayer of $0.35 \mu \mathrm{m}$ and a Si-based interlayer of $0.6 \mu \mathrm{m}$. The elastic modulus of the H-DLC coating is $151 \pm 3 \mathrm{GPa}$ and the hardness is $18.7 \pm 0.5 \mathrm{GPa}$. The oil has a viscosity of $76.6 \mathrm{~mm}^{2} / \mathrm{s}$ at $40{ }^{\circ} \mathrm{C}$ and $12.3 \mathrm{~mm}^{2} / \mathrm{s}$ at $100{ }^{\circ} \mathrm{C}$. The tests were performed at an elevated temperature of $100{ }^{\circ} \mathrm{C}$ to develop the ZDDP tribofilm on the DLC coating. The normal load was set to $10 \mathrm{~N}$, which corresponds to $1 \mathrm{GPa}$ of maximum initial Hertzian contact pressure. A frequency of 10 $\mathrm{Hz}$ and a stroke length of $5 \mathrm{~mm}$ were set, resulting in an average contact velocity of $0.1 \mathrm{~m} / \mathrm{s}$. The durations of the tests were 15,000 and 216,000 cycles. After the tribological tests the discs were gently rinsed with a solvent (n-heptane) to remove the excess oil, while retaining the adsorbed tribofilms on the surface.

In order to analyse and determine the mechanical properties of the ZDDP tribofilms on the DLC coatings, nano-indentations were carried out with a Berkovich tip using a Nano-indenter (G200, Keysight Technologies, CA, USA). Using the Continuous Stiffness Measurement (CSM) mode, the loadings of the load-displacement curves were recorded. The nanoindentation measurements were performed on the surface of the tribofilm at several locations inside the wear track so as to achieve the most representative results. In order to avoid any influence of the substrate, the indentation depth should be set to approximately $10 \%$ of the thickness of the thin films [18]. Therefore, the indentation depth for was set to $20 \mathrm{~nm}$, due to the thickness of the ZDDP tribofilm being 175-200 nm, measured on the same films using an AFM (AFM CP-II, Brucker, CA, USA) [8].

To verify and understand the nano-indentation data in more detail, the AFM force modulation mode (FMM), which provides local contrast for the mechanical stiffness properties of the surface, was used to evaluate the local mechanical behaviour on the surface of the ZDDP tribofilms. The FMM mode is based on the contact AFM mode; the feedback loop maintains a constant cantilever deflection, i.e. a constant force on the sample. The variation in the cantilever's deflection amplitude at the frequency of modulation is a measure of the relative stiffness of the surface. Therefore, the FMM imaging makes it possible to study the local mechanical properties of the surface, i.e. the relative differences in surface elasticity at nanoscale resolution [19]. The scan size of the FMM images, where the stiffness difference is shown by the brightness on the surface of the ZDDP tribofilms, was $5 \times 5 \mu \mathrm{m}^{2}$. 
This peer reviewed manuscript has been accepted for publications to the Tribology Letters. Cite this article as: Luis Bartolomé, Eva Oblak, Mitjan Kalin, Sliding evolution of the mechanical behaviour of zinc dialkyldithiophosphate tribofilms on diamond-like carbon coatings Tribology letters 62 (2016) 0109. DOI: https://doi.org/ 10.1007/s11249-016-0666-y

\subsection{Numerical methods}

In order to numerically determine the mechanical properties of the ZDDP tribofilms, an algorithm was defined (Fig. 1). This algorithm consists of a forward algorithm and an inverse algorithm, which should be performed sequentially.
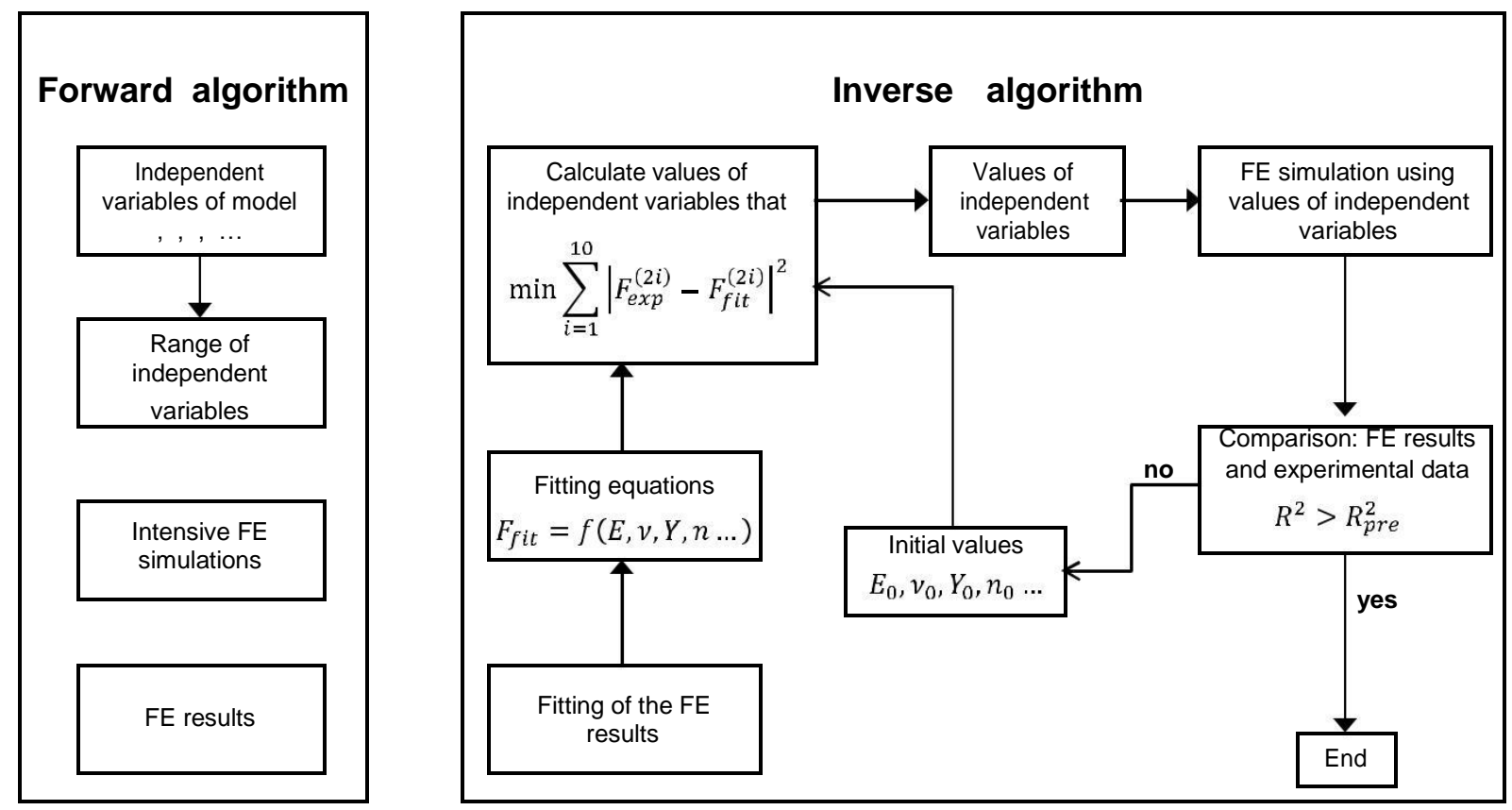

Figure 1: Algorithm for determining the mechanical properties of ZDDP tribofilms.

The forward algorithm begins by establishing the independent variables or material properties of the constitutive model under consideration. When the independent variables are established, a range of values is set for each independent variable, with enough intermediate values to provide a good fitting. Then intensive FE simulations were performed using all the possible combinations of the different values for the independent variables. During the numerical calculus, ten depths, from $2 \mathrm{~nm}$ to $20 \mathrm{~nm}$ in steps of $2 \mathrm{~nm}$, were defined along the loading curve. The numerical results of the loads at the chosen indentation depths were saved in order to be used in the fitting process during the inverse algorithm. The inverse algorithm begins by fitting the FE results using polynomial equations for each specific point of the loading curve. Using these equations, the values of the independent variables that minimize the difference between the experimental data and the values from the fitting equations are calculated for all the specific points at the same time. These calculated values for the independent variables are implemented into the FE nano-indentation simulation again and the results of the loaddisplacement curve are compared with the experimental data. The values of the independent variables that show the best correlation with the experimental data are chosen as a solution because the solution of the nano-indentation at moderate depths is unique according to Chen et al. [20]. This algorithm is explained in more detail elsewhere [10].

The numerical simulations of the nano-indentations for the algorithms were performed by means of the finite element method and the FE analysis was implemented using the commercial package Abaqus (version 6.13-4, ABAQUS Inc.). The nano-indentations were simulated using an axisymmetric model and these simulations were displacement-controlled. 
This peer reviewed manuscript has been accepted for publications to the Tribology Letters. Cite this article as: Luis Bartolomé, Eva Oblak, Mitjan Kalin, Sliding evolution of the mechanical behaviour of zinc dialkyldithiophosphate tribofilms on diamond-like carbon coatings Tribology letters 62 (2016) 0109. DOI: https://doi.org/ 10.1007/s11249-016-0666-y

The ZDDP tribofilms were modelled as a flat body and the Berkovich indenter was simulated as a cone with a semi-angle of $70.3^{\circ}$, which gives the same area to depth relationship as a pyramidal Berkovich indenter [18]. In all the simulations, 4-node, bilinear, axisymmetric, quadrilateral, hybrid, constant pressure $(\mathrm{CAX} 4 \mathrm{H})$ elements were used to model the ZDDP tribofilm and the node bilinear axisymmetric quadrilateral (CAX4) elements were used to model the Berkovich indenter. The mechanical properties of the ZDDP tribofilm were changed depending on the selected constitutive model, and the indenter $(E=1100 \mathrm{GPa}, v=0.07)$ was considered as an isotropic, linear elastic material. The model consists of 37,120 elements for the ZDDP tribofilm and 20,160 elements for the indenter. The normal displacement at the bottom of the ZDDP model was fixed. The reaction forces in the normal direction were recorded at every $2 \mathrm{~nm}$ of indentation depth between $2 \mathrm{~nm}$ and $20 \mathrm{~nm}$ for a comparison with the experimental data.

\section{RESULTS}

\subsection{Experimental results}

The results of the experimental nano-indentations on the ZDDP tribofilms for the DLC coatings after 15,000 cycles show two distinctly different behaviours. These two behaviours are very consistently grouped in a clear mechanical response, as the experimental loaddisplacement curves show in Fig. 2. But due to the experimental measurements, both mechanical responses exhibit some scatter for both components of the ZDDP tribofilms on the DLC coatings. Therefore, an average for the experimental load-displacement curves was calculated for both components (see the dotted lines in Fig. 2), which are named as harder and softer components after 15,000 cycles. These two average curves, were used to compare with the numerical results of the FE simulations.

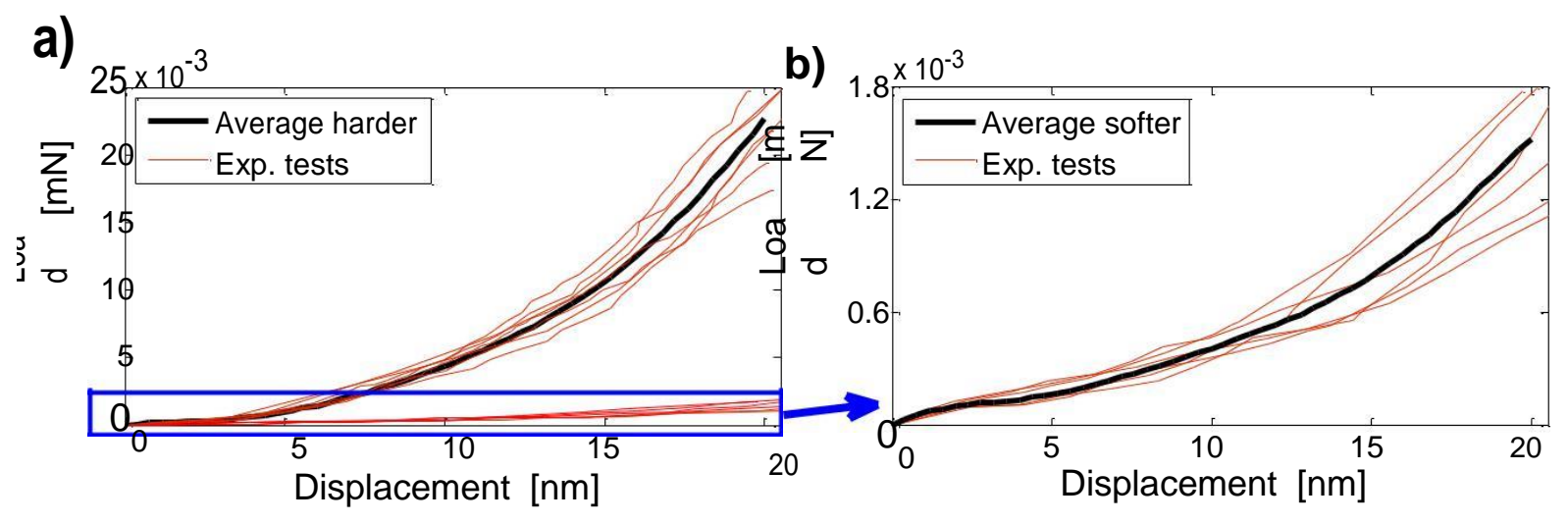

Figure 2: (a)Experimental displacement-load curves of the nano-indentations on the ZDDP tribofilms obtained at 15,000 cycles showing two distinctive mechanical responses and (b)augmentation for softer component.

The results of the experimental nano-indentations on the ZDDP tribofilms for the DLC coatings after 216,000 cycles are shown in Fig. 3. In this case, the experimental loaddisplacement curves show three distinctly different behaviours, which are also very consistently grouped in a clear mechanical response and also exhibit some scatter for the 
This peer reviewed manuscript has been accepted for publications to the Tribology Letters. Cite this article as: Luis Bartolomé, Eva Oblak, Mitjan Kalin, Sliding evolution of the mechanical behaviour of zinc dialkyldithiophosphate tribofilms on diamond-like carbon coatings Tribology letters 62 (2016) 01-09. DOI: https://doi.org/ 10.1007/s11249-016-0666-y

components of the ZDDP tribofilms. The averages for the experimental load-displacement curves were calculated for the three components (see the dotted lines in Fig. 3). In this case, after 216,000 cycles, these three components are referred as harder, medium and softer through this work. These three average curves for each component were used to compare with the numerical results of the FE simulations.

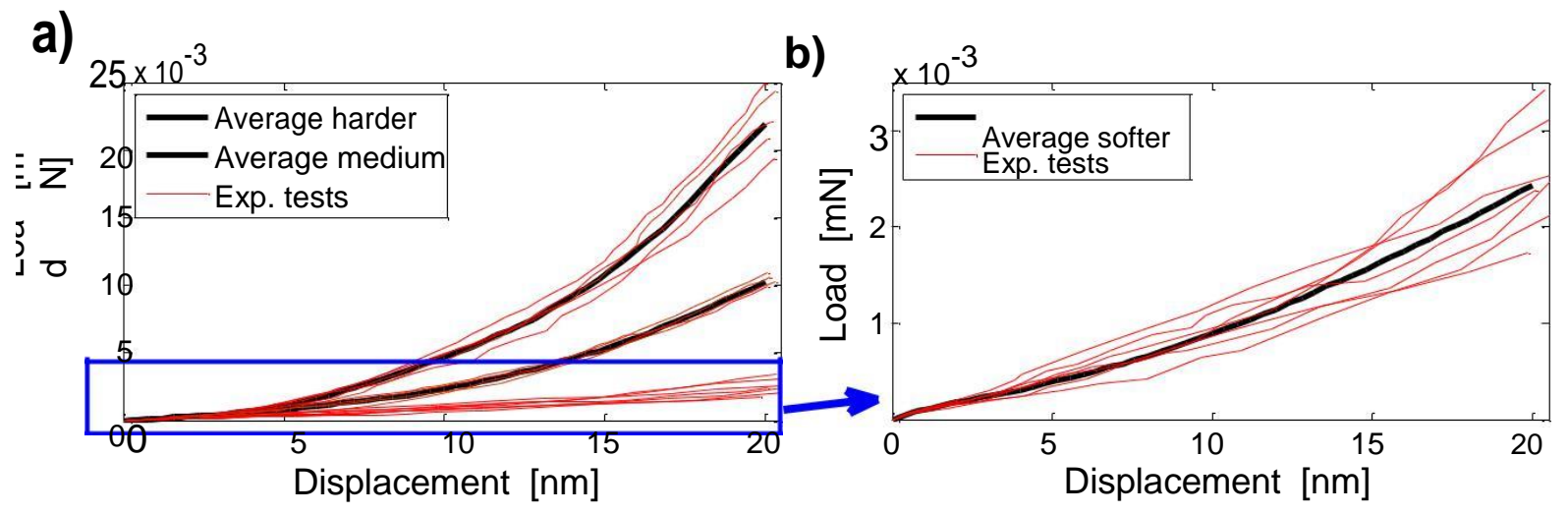

Figure 3: (a) Experimental displacement-load curves of the nano-indentations on the ZDDP tribofilms obtained at 216,000 cycles showing three distinctive mechanical responses and (b)augmentation for softer component.

\subsection{Computational results}

\subsubsection{Harder component}

The mechanical behaviour of the harder components of the ZDDP tribofilms for both test sliding distances (15,000 and 216,000 cycles) is modelled more accurately using an elastic plastic model obeying a power law [10]. Fig. 4 shows the correlations between the experimental data and the numerical solutions.
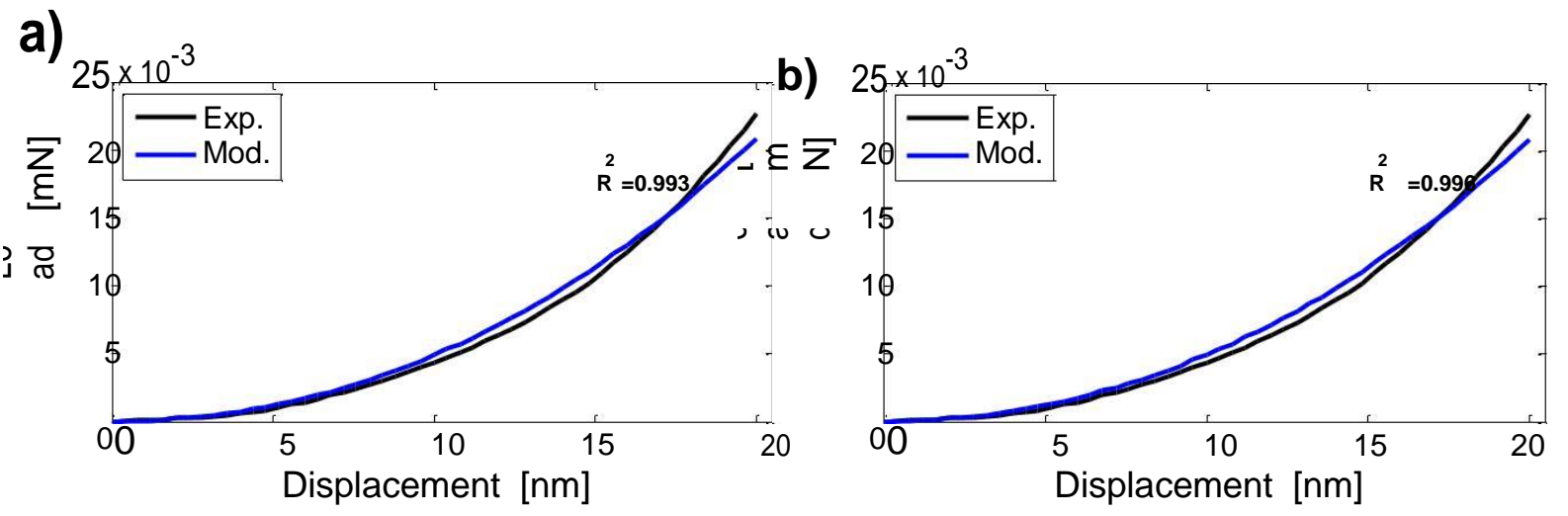

Figure 4: Comparison between the experimental nano-indentation data and the numerical displacementload results using the elastic plastic model obeying a power law for the harder component of the ZDDP tribofilms obtained at (a)15,000 cycles and (b)216,000 cycles. 
This peer reviewed manuscript has been accepted for publications to the Tribology Letters. Cite this article as: Luis Bartolomé, Eva Oblak, Mitjan Kalin, Sliding evolution of the mechanical behaviour of zinc dialkyldithiophosphate tribofilms on diamond-like carbon coatings Tribology letters 62 (2016) 01-09. DOI: https://doi.org/ 10.1007/s11249-016-0666-y

\subsubsection{Medium component}

The mechanical behaviour of the medium component of the ZDDP tribofilms after 216,000 cycles) is modelled more accurately using an elastic-plastic model obeying a power law. Fig. 5 shows the correlations between the experimental data and the numerical solutions.

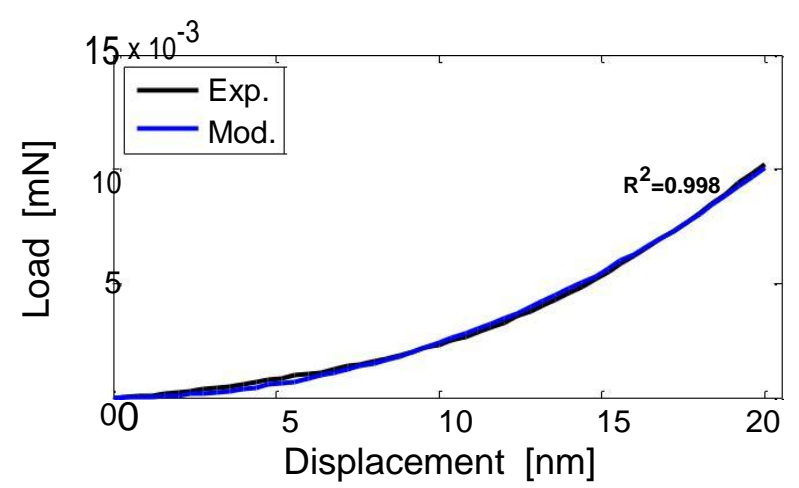

Figure 5: Comparison between the experimental nano-indentation data and the numerical displacementload results using the elastic plastic model obeying a power law for the medium component of the ZDDP tribofilms obtained at 216,000 cycles.

\subsubsection{Softer component}

In the case of the softer components of the ZDDP tribofilms for both sliding distances $(15,000$ and 216,000 cycles), the mechanical behaviour is modelled more accurately using a neoHookean hyperelastic model with compressibility [10]. Fig. 6 shows the correlations between the experimental data and the numerical solutions using a neo-Hookean hyperelastic model. The obtained values for the parameters of the neo-Hookean hyperelastic model were $C_{10}=0.39 \pm 0.04 \mathrm{GPa}$ and $D_{1}=3.8 \pm 0.6 \mathrm{GPa}^{-1}$ after 15,000 cycles and $C_{10}=2.1 \pm 0.2 \mathrm{GPa}$ and $D_{1}=0.7 \pm 0.08 \mathrm{GPa}^{-1}$ after 216,000 cycles.

a)

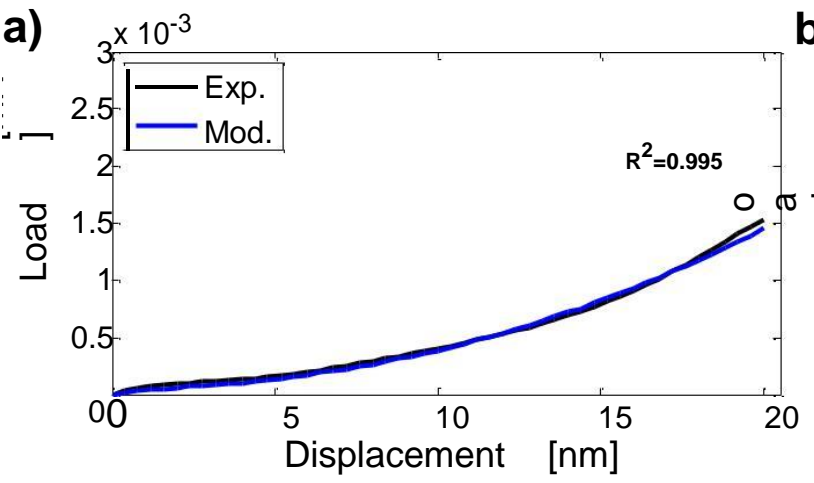

b)

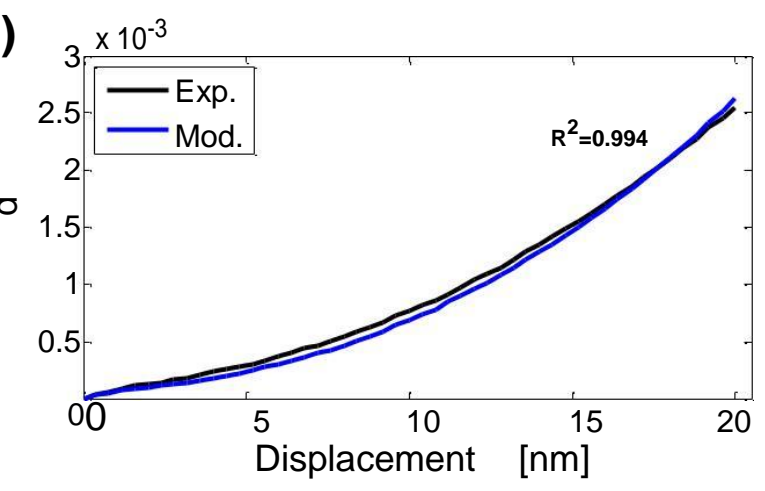

Figure 6: Comparison between the experimental nano-indentation data and the numerical displacementload results using the neo-Hookean hyperelastic model for the softer component of the ZDDP tribofilms obtained at (a)15,000 cycles and (b)216,000 cycles.

However, in order to easily compare the mechanical properties among the different components of the ZDDP tribofilms, the mechanical behaviour was also fitted using the elastic-plastic model obeying a power law. Fig. 7 shows the correlations between the 
This peer reviewed manuscript has been accepted for publications to the Tribology Letters. Cite this article as: Luis Bartolomé, Eva Oblak, Mitjan Kalin, Sliding evolution of the mechanical behaviour of zinc dialkyldithiophosphate tribofilms on diamond-like carbon coatings Tribology letters 62 (2016) 0109. DOI: https://doi.org/ 10.1007/s11249-016-0666-y

experimental data and the numerical solutions using an elastic plastic model obeying a power law.
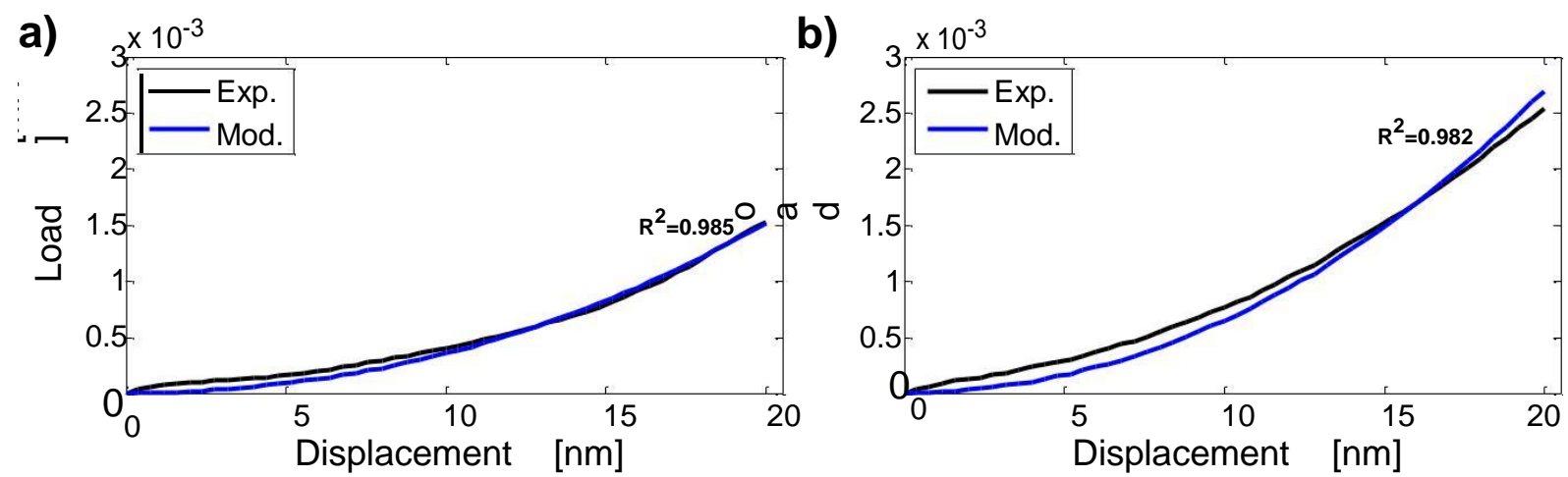

Figure 7: Comparison between the experimental nano-indentation data and the numerical displacementload results using the elastic plastic model obeying a power law for the softer component of the ZDDP tribofilms obtained at (a)15,000 cycles and (b)216,000 cycles.

\section{DISCUSION}

The experimental results of the nano-indentations (Fig. 2 and Fig. 3) clearly show that the ZDDP tribofilms have a non-homogeneous structure after both sliding times. These nonhomogeneous structured tribofilms agree with the literature about ZDDP tribofilms on steel [2, 3] and on DLC coatings [6], where a non-homogeneous structure is commonly observed. The experimental results of the nano-indentations clearly show that the ZDDP tribofilms after 15,000 cycles have a dual structure where two different mechanical responses are observed (see Fig. 2). This fact indicates that the ZDDP tribofilms on the DLC coatings after a small number of tribological cycles consist of two distinctively different material components. This was found in our previous work [10] and is the subject of on-going investigations [7, 8]. Moreover this fact is in agreement with the literature about ZDDP tribofilms on DLC [6], where structured ZDDP tribofilms with different kinds of sulphites and phosphates are found. However, the experimental results for the ZDDP tribofilms after 216,000 cycles show a structure that has three components with three different mechanical responses (see Fig. 3). Therefore, the ZDDP tribofilms on the DLC coatings cannot be considered as a homogeneous material with a unique mechanical behaviour, but as a structured material with different components that exhibit very different mechanical behaviours, which should take into account when the ZDDP tribofilms are modelled.

In this work, the structure of the ZDDP tribofilms after both tested sliding times were additionally proved using AFM topography and the force modulation mode (FMM), which provides local contrast in the mechanical stiffness properties of the surface. Fig. 8a shows a topography image of a ZDDP tribofilm after 15,000 cycles and Fig. $8 \mathrm{~b}$ shows the corresponding image using the FMM. In the case of the ZDDP tribofilm after 216,000 cycles, Fig. 9a shows the topography image and Fig. 9b shows the corresponding image using the FMM. In Fig. $8 b$ and Fig. $9 b$ the bright areas correspond to stiffer areas of the ZDDP 
This peer reviewed manuscript has been accepted for publications to the Tribology Letters. Cite this article as: Luis Bartolomé, Eva Oblak, Mitjan Kalin, Sliding evolution of the mechanical behaviour of zinc dialkyldithiophosphate tribofilms on diamond-like carbon coatings Tribology letters 62 (2016) 01-09. DOI: https://doi.org/ 10.1007/s11249-016-0666-y

tribofilm surface, while the dark areas represent the more elastic and more compliant components of the surface [19]. Focusing on the highlighted zones, i.e. the white contours, in Fig. 8, it is clear that these zones present dark and bright areas. The same difference between dark and bright areas is shown for the ZDDP tribofilms after 216,000 cycles (see the zones highlighted by the white contours in Fig. 9). However, in this case, different intensities can be also observed in the bright areas. All of this further confirms that the structure of ZDDP tribofilms becomes more complex over the sliding time changing from a dual structure to even a three-component structure.
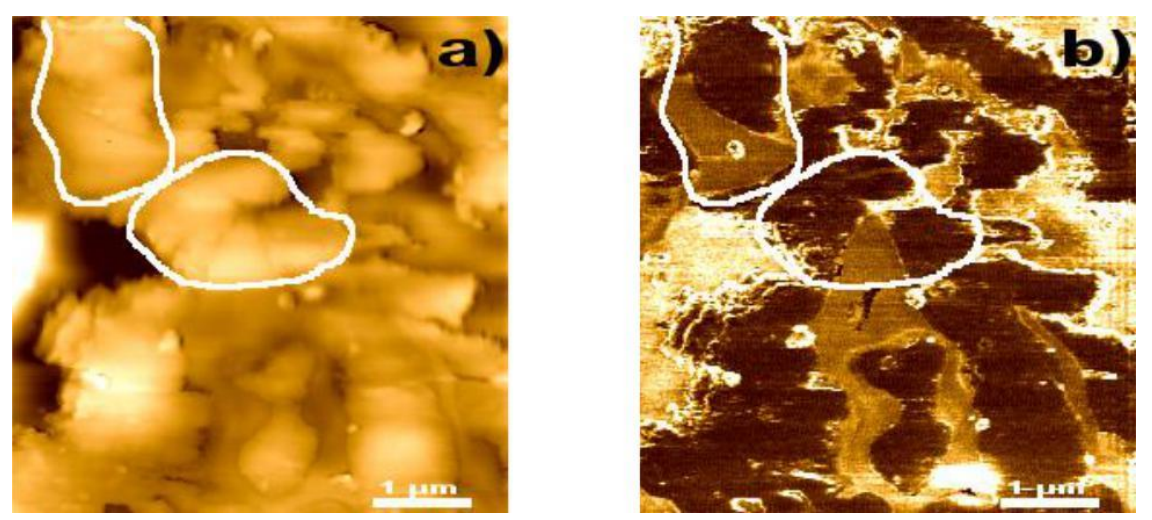

Figure 7: Images of ZDDP tribofilms on DLC coating at 15,000 cycles: (a)topography and (b)the corresponding stiffness map using FMM mode.
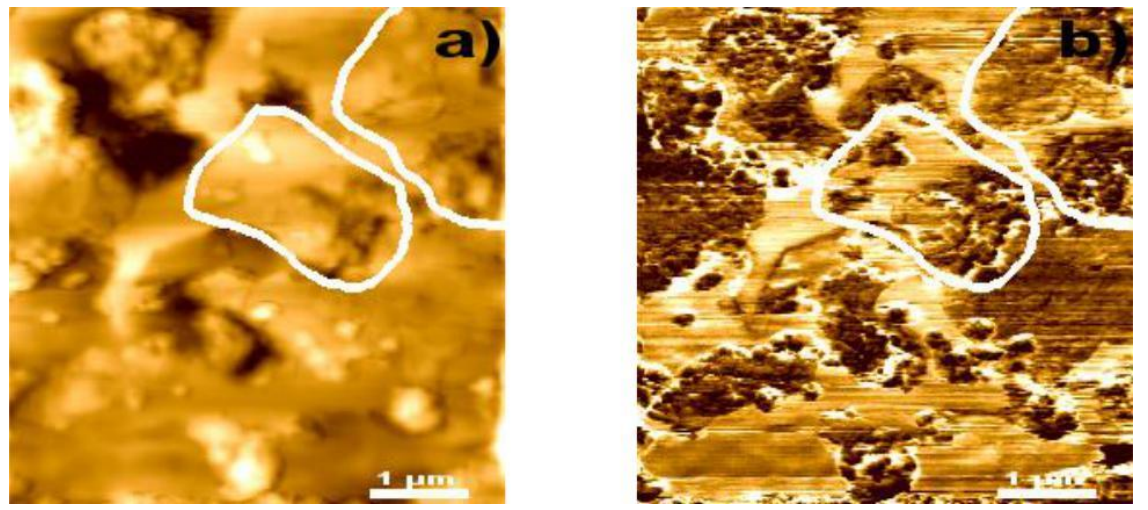

Figure 8: Images of ZDDP tribofilms on DLC coating at 216,000 cycles: (a)topography and (b)the corresponding stiffness map using FMM mode.

The mechanical behaviours of the harder components (Fig. 4) for both test durations and the medium component (Fig. 5) of the ZDDP tribofilms on the DLC coatings are predicted more accurately using an elastic-plastic model obeying a power law due to this constitutive model taking into account the plastic deformation during the nano-indentations. However the mechanical behaviours of the softer components (Fig. 6) for both test durations are predicted more accurately using the neo-Hookean hyperelastic model with compressibility. More details about the analysis of the various considered models, i.e. elastic, elastic-perfectly plastic, elastic-plastic with hardening, and hyperplastic, can be found in an earlier work [10]. However, in order to compare the mechanical properties of the different components, the elastic-plastic model obeying a power law was also used to fit the mechanical behaviour of the softer components for both test durations. The values of the mechanical properties are 
This peer reviewed manuscript has been accepted for publications to the Tribology Letters. Cite this article as: Luis Bartolomé, Eva Oblak, Mitjan Kalin, Sliding evolution of the mechanical behaviour of zinc dialkyldithiophosphate tribofilms on diamond-like carbon coatings Tribology letters 62 (2016) 0109. DOI: https://doi.org/ 10.1007/s11249-016-0666-y

summed up in Table 1 for every component of the ZDDP tribofilms when the elastic-plastic model obeying a power law was used. Therefore, the mechanical modelling of the ZDDP tribofilms on DLC coatings becomes a complex task because the ZDDP tribofilms exhibit non-homogenous mechanical properties that also change over the time of the contact. The errors for the mechanical properties were calculated by partially differentiating the fitting equations with respect to the independent variables and considering the largest error from among all the results obtained under the different loads.

Table 1: Mechanical parameters for the components of the ZDDP tribofilms on the DLC coatings.

\begin{tabular}{lccccc}
\hline $\begin{array}{l}\text { Component of } \\
\text { ZDDP tribofilm }\end{array}$ & Cycles & $\begin{array}{c}\text { Elastic mod. } \\
\boldsymbol{E}[\mathrm{GPa}]\end{array}$ & $\begin{array}{c}\text { Poisson's ratio } \\
\mathbf{N}\end{array}$ & $\begin{array}{c}\text { Yield stress } \\
\boldsymbol{Y}[\mathrm{GPa}]\end{array}$ & $\begin{array}{c}\text { Hardening exp. } \\
\boldsymbol{n}\end{array}$ \\
\hline \multirow{2}{*}{ Harder } & 15000 & $26 \pm 1$ & $0.41 \pm 0.03$ & 1.55 & $0.52 \pm 0.05$ \\
& 216000 & $25 \pm 1$ & $0.40 \pm 0.03$ & 1.62 & $0.48 \pm 0.05$ \\
\multirow{2}{*}{ Medium } & 15000 & - & - & - & - \\
& 216000 & $11.7 \pm 0.6$ & $0.39 \pm 0.03$ & 0.94 & $0.055 \pm 0.004$ \\
\multirow{2}{*}{ Softer } & 15000 & $1.65 \pm 0.09$ & $0.42 \pm 0.02$ & 0.144 & $0.21 \pm 0.02$ \\
& 216000 & $3.5 \pm 0.2$ & $0.39 \pm 0.02$ & 0.17 & $0.22 \pm 0.02$ \\
\hline
\end{tabular}

Regarding the harder components of the ZDDP tribofilms, it was found that the mechanical properties of the ZDDP tribofilms for both tested sliding times remain virtually unaltered over the period of the sliding (see Table 1). The harder component of the ZDDP tribofilms enhances the mechanical strength of the tribofilms, as was suggested by earlier studies of ZDDP tribofilms on steel [3] and DLC coatings [6]. This unaltered mechanical behaviour over time indicates that chemically this component occurs since the beginning of the contact and it is maintained over the sliding time.

The medium component of the ZDDP tribofilms is only observed after 216,000 cycles. Therefore this component appears to have slower chemical kinetics than the other components. As the harder component, the medium component could also provide strength to the tribofilms. This medium component clearly exhibits less stiff mechanical behaviour than the harder component and clearly also a stiffer behaviour than the softer component. The mechanical properties of the medium components can be compared with the other components in Table 1. It can be speculated that this medium component could be chemically similar or the same as the harder component, but initiated at a later time or it could be a new component with different kinetics and result from intermediate reaction products. However, a proof of this fact requires detailed chemical analyses.

Concerning the softer components of the ZDDP tribofilms, a slight stiffening is found over time (see Table 1). The softer components are presumably composed of precipitates from the degradation process and wear [3], providing lubrication in the contact [21]. This slight stiffening of the softer component could be explained mechanically even though it could be based on chemical or thermal effects. The mechanical explanation would be related to the fact that the generation of the debris and the degradation aggregates is a process over the sliding time with its own kinetics. Therefore, at the beginning of the sliding contact, the debris start to be created intensively because the wear is more severe and the degradation also starts to occur. When the sliding time passes, these debris and aggregates, which are compressible 
This peer reviewed manuscript has been accepted for publications to the Tribology Letters. Cite this article as: Luis Bartolomé, Eva Oblak, Mitjan Kalin, Sliding evolution of the mechanical behaviour of zinc dialkyldithiophosphate tribofilms on diamond-like carbon coatings Tribology letters 62 (2016) 0109. DOI: https://doi.org/ 10.1007/s11249-016-0666-y

hyperelastic materials as explained above, suffer permanent compression deformation due to the cyclic contact, and then this component gets stiffer over time [Fig. 10].
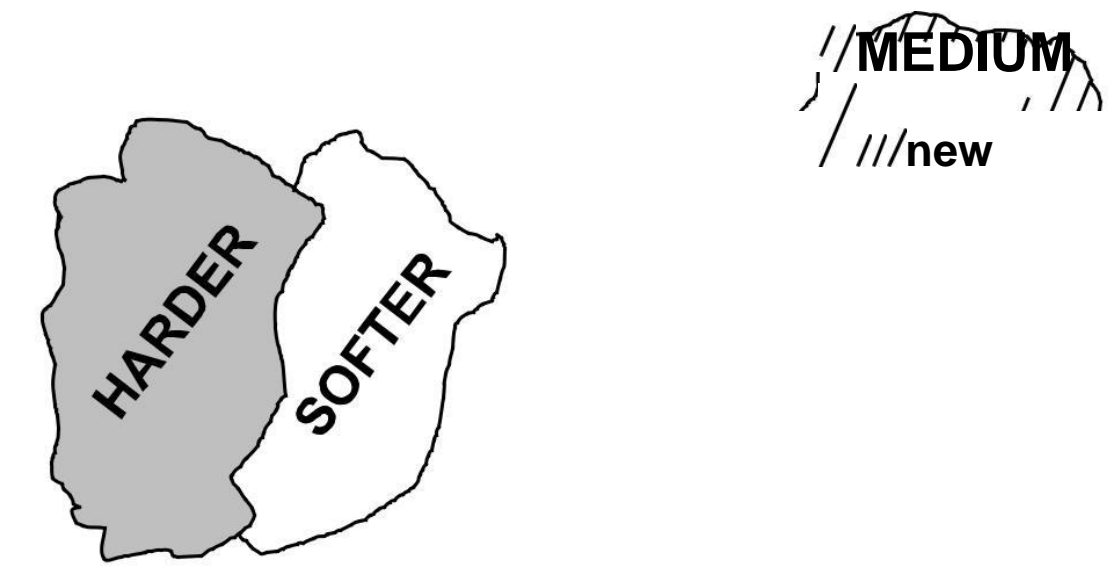

Sliding time

Figure 9: Schematic representation of the ZDDP tribofilm structure over the time (axial view).

In summary, in view of all the results from the nano-mechanical and finite element analyses, the ZDDP tribofilms on the DLC coatings cannot be described by a single, unique, mechanical response, but rather the ZDDP tribofilms have multiple components that exhibit different mechanical behaviours. Therefore the ZDDP tribofilms should be considered as structured materials where the mechanical properties of the components could differ by as much as an order of magnitude (see Table 1). The mechanical behaviour of these multistructured ZDDP tribofilms on DLC coatings is relatively complex, depending on the type of DLC coatings, additives and sliding distances [7, 8], but the multi-structure of the ZDDP tribofilms also evolves over the sliding time. Therefore, the mechanical modelling of the ZDDP tribofilms on DLC coatings becomes a complex task because they exhibit nonhomogenous mechanical properties that also change over time. However the current results show that a nano-mechanical analysis coupled with FEM can provide important findings about how the ZDDP tribofilms are structured during the sliding contact.

\section{CONCLUSIONS}

The sliding evolution of the ZDDP tribofilms on DLC coatings presents the following features:

- The structure of the ZDDP tribofilms on DLC coatings changes during the sliding time. After 15,000 cycles, it is found a dual structure with a softer and a harder component, but, after 216,000 cycles, the ZDDP tribofilms have three different components: harder, medium and softer.

- The harder component of the ZDDP tribofilms on the DLC coatings remains unchanged in its mechanical behaviour during the sliding time.

- The softer component of the ZDDP tribofilms on the DLC coatings suffers slight stiffening during the sliding time. 
This peer reviewed manuscript has been accepted for publications to the Tribology Letters. Cite this article as: Luis Bartolomé, Eva Oblak, Mitjan Kalin, Sliding evolution of the mechanical behaviour of zinc dialkyldithiophosphate tribofilms on diamond-like carbon coatings Tribology letters 62 (2016) 0109. DOI: https://doi.org/ 10.1007/s11249-016-0666-y

- The mechanical behaviour of the harder and the medium components are predicted accurately using the elastic-plastic model obeying a power law for both sliding times of the tribological tests, i.e. 15,000 and 216,000 cycles.

- The mechanical behaviour of the softer component is predicted accurately using the neo-Hookean hyperelastic model with compressibility for both sliding times of the tribological tests, i.e. 15,000 and 216,000 cycles.

- The ZDDP tribofilms on the DLC coatings cannot be considered as a homogeneous material with a steady and unique mechanical behaviour, but as a structured material with different components that exhibit very different mechanical behaviours, which can even change over the time period of sliding.

\section{ACKNOWLEDGEMENTS}

This study was funded by the FP7 program through the Marie Curie Initial Training Network (MC-ITN) entitled "ENTICE - Engineering Tribochemistry and Interfaces with a Focus on the Internal Combustion Engine" [290077] and was carried out at University of Ljubljana. The authors would like to thank to all ENTICE partners for kind discussions on the topic and the methodology.

\section{REFERENCES}

[1] Kalin, M., Velkavrh, I., Vižintin, J., Ožbolt L.: Review of boundary lubrication mechanisms of DLC coatings used in mechanical applications. Meccanica. 43, 623-637 (2008)

[2] Spikes, H.: The history and mechanisms of ZDDP. Tribol. Lett. 17, 469-489 (2004)

[3] Bec, S., Tonck, A., Georges, J.M., Coy, R.C., Bell, J.C., Roper, G.W.: Relationship between mechanical properties and structures of zinc dithiophosphate anti-wear films. Proc Royal Soc. Lond. A Math., Phys. Eng. Sci. 455, 4181-4203 (1999)

[4] Vengudusamy, B., Green, J.H., Lamb, G.D., Spikes, H.A.: Tribological properties of tribofilms formed from ZDDP in DLC/DLC and DLC/steel contacts. Tribol. Int. 44, 165-174 (2011)

[5] Equey, S., Roos, S., Mueller, U., Hauert, R., Spencer, N.D., Crockett, R.: Reactions of zinc-free anti-wear additives in DLC/DLC and steel/steel contacts. Tribol. Int. 41, 1090-1096 (2008)

[6] Vengudusamy, B., Green, J., Lamb, G., Spikes, H.: Durability of ZDDP Tribofilms Formed in DLC/DLC Contacts. Tribol. Lett. 51, 469-478 (2013)

[7] Oblak, E., Kalin, M.: Relationship between the nano-scale topographical and mechanical properties of tribochemical films on DLC coatings and their macroscopic friction behavior. Tribol. Lett. (2015). doi:10.1007/s11249-015-0575-5 
This peer reviewed manuscript has been accepted for publications to the Tribology Letters. Cite this article as: Luis Bartolomé, Eva Oblak, Mitjan Kalin, Sliding evolution of the mechanical behaviour of zinc dialkyldithiophosphate tribofilms on diamond-like carbon coatings Tribology letters 62 (2016) 0109. DOI: https://doi.org/ 10.1007/s11249-016-0666-y

[8] Kalin, M., Oblak, E., Akbari, S.: Evolution of the nano-scale mechanical properties of tribofilms formed from low- and high-SAPS oils and ZDDP on DLC coatings and steel. Tribol. Int. 96, 43-56 (2016)

[9] Onodera, T., Kuriaki, T., Morita, Y., Suzuki, A., Koyama, M., Tsuboi, H., Hatakeyamaa, N., Endoua, A., Takabac, H., Del Carpioa, C.A., Kubod, M., Minfraye, C., Martine, J.M., Miyamotoa, A.: Influence of nanometer scale film structure of ZDDP tribofilm on its mechanical properties: A computational chemistry study. Appl. Surf. Sci. 256, 976-979 (2009)

[10] Bartolomé, L., Oblak, E., Kalin, M.: Mechanical behaviour and constitutive models of ZDDP tribofilms on DLC coatings using nano-indentation data and finite element modelling. Tribol. Int. 95, 19-26 (2016)

[11] Heinrich, C., Waas, A.M., Wineman, A.S.: Determination of material properties using nanoindentation and multiple indenter tips. Int. J. Solids Struct. 46, 364-376 (2009)

[12] Oliver, W.C., Pharr, G.M.: An improved technique for determining hardness and elastic modulus using load and displacement sensing indentation experiments. J. Mater. Res. 7, 1564$1583(1992)$

[13] Cheng, Y., Cheng, C.. Scaling, dimensional analysis, and indentation measurements. Mater. Sci. Eng. R 44, 91-149 (2004)

[14] Chen, X., Yan, J., Karlsson, A.M.: On the determination of residual stress and mechanical properties by indentation. Mater. Sci. Eng. A. 416, 139-149 (2006)

[15] Zheng, Y.P., Choi, A.P.C., Ling, H.Y., Huang, Y.P.: Simultaneous estimation of Poisson's ratio and Young's modulus using a single indentation: a finite element study. Meas. Sci. Technol. 20, 1-9 (2009)

[16] Kuo, J., Huang, I.: Extraction of plastic properties of aluminum single crystal using Berkovich indentation. Mater. Trans. 51, 2104-2108 (2010)

[17] Abdullah Tasdemir, H., Wakayama, M., Tokoroyama, T., Kousaka, H., Umehara, N., Mabuchi, Y., Higuchib, T.: The effect of oil temperature and additive concentration on the wear of non-hydrogenated DLC coating. Tribol. Int. 77, 65-71 (2014)

[18] Fischer-Cripps, A.C.: Nanoindentantion. Springer Science+Business Media LLC, New York (2011)

[19] Li, F., Thompson, G.E., Newman, R.C.: Force modulation atomic force microscopy: background, development and application to electrodeposited cerium oxide films. Appl. Surf. Sci. 126, 21-33 (1998)

[20] Chen, X., Ogasawara, N., Zhao, M., Chiba, N.: On the uniqueness of measuring elastoplastic properties from indentation: The indistinguishable mystical materials. J. Mech. Phys. Solids 55, 1618-1660 (2007) 
This peer reviewed manuscript has been accepted for publications to the Tribology Letters. Cite this article as: Luis Bartolomé, Eva Oblak, Mitjan Kalin, Sliding evolution of the mechanical behaviour of zinc dialkyldithiophosphate tribofilms on diamond-like carbon coatings Tribology letters 62 (2016) 01-09. DOI: https://doi.org/ 10.1007/s11249-016-0666-y

[21] Ye, J., Kano, M., Yasuda, Y.: Evaluation of local mechanical properties in depth in modtc/zddp and zddp tribochemical reacted films using nanoindentation. Tribol. Lett. 13, 4147 (2002) 